\title{
Inclusión de Ensilado de Residuos de Trucha en el Alimento de Cerdos y su Efecto en el Rendimiento Productivo y Sabor de la Carne
}

\author{
Effect of the Inclusion of Silage of Trout Residues in Pigs Feed and Its \\ Effect on the Productive Performance and the Taste of Meat
Víctor Calderón-Quispe', Marisol Churacutipa-Mamani², Alberto Salas ${ }^{3}$, Maritza Barriga-Sánchez ${ }^{3,4}$, Marcelino J. Araníbar ${ }^{1}$

\section{ResUmen}

\begin{abstract}
Con la finalidad de determinar el efecto del nivel de inclusión de ensilado de residuos de trucha (ERT) en el alimento sobre el peso vivo, consumo de alimento y conversión alimenticia en cerdos, así como en el sabor de su carne, se plantearon tres tratamientos: un testigo (T1, 0\%) y dos con niveles de $5 \%$ (T2) y $10 \%$ (T3) de inclusión de ERT en la ración, donde cada tratamiento se hizo con cinco réplicas. Se utilizaron 30 cerdos machos enteros de 45 días de edad (York-Duroc-Landrace). Los alimentos de crecimiento y engorde fueron similares en proteína (PB) y energía metabolizable (EM) (crecimiento: $16.0 \%$ PB y $3.20 \mathrm{Mcal} / \mathrm{kg}$ EM y engorde: $13.0 \%$ PB y $3.20 \mathrm{Mcal} / \mathrm{kg}$ EM) y fueron administrados ad libitum durante 98 días. Se determinó el peso vivo, el consumo de alimento y la conversión alimenticia a los 56 y 98 días. Al final se realizó una prueba sensorial para evaluar el sabor de la carne. A los 98 días, los resultados indicaron que los tratamientos T1, T2 y T3 no afectaron el peso vivo $(69.8,81.3$ y $86.4 \mathrm{~kg}$, respectivamente), ni el consumo de alimento $(1.30,1.50$ y $1.54 \mathrm{~kg} /$ día, respectivamente). Sin embargo, la conversión alimenticia fue mejor con el incremento del ERT en la ración $(2.25,2.15$ y 2.04 para T1, $\mathrm{T} 2$ y T3, respectivamente, $\mathrm{p}=0.012$ ). Las calificaciones de la prueba sensorial indicaron que la inclusión de ERT en el alimento no afectó el sabor de la carne. Se concluye que hasta 10\% de inclusión de ERT en el alimento mejora la conversión alimenticia y no afecta el sabor de la carne de los cerdos.
\end{abstract}

Palabras clave: cerdos, ensilado de trucha, rendimiento productivo, alimentación

\footnotetext{
${ }^{1}$ Facultad de Medicina Veterinaria y Zootecnia, Universidad Nacional del Altiplano, Puno, Perú

${ }^{2}$ Arapa San Pedro San Pablo SAC, Puno, Perú

${ }^{3}$ Dirección de Investigación, Desarrollo, Innovación y Transferencia Tecnológica, Instituto Tecnológico de la Producción, Lima, Perú

${ }^{4}$ E-mail: mbarriga@itp.gob.pe
} 
The study aimed to determine the effect of the level of inclusion of silage from trout residues (ERT) in concentrate feed on body weight, feed intake, feed conversion of pigs and taste of pig meat. Three treatments were applied: a control (T1, $0 \%)$ and two treated groups with 5\% (T2) and 10\% (T3) of ERT in the ration, with five replicates per treatment. Thirty non-castrated male pigs (York-Duroc-Landrace) of 45 days of age were used. Feeds for the growing and finishing phase were formulated to be similar in protein (CP) and energy (ME) content (growing: 16.0\% CP and 3.20 Mcal ME/kg and finishing: 13.0\% $\mathrm{CP}$ and $3.20 \mathrm{Mcal} \mathrm{ME} / \mathrm{kg}$ ) and were administered ad libitum during 98 days. Body weight, feed intake and feed conversion rate were determined at 56 and 98 days. In addition, a sensory test of meat was carried out at the end of the study to evaluate the taste of the meat. The results at day 98 showed no differences between treatments on body weight $(69.8,81.3$ and $86.4 \mathrm{~kg}$ for T1, T2 and T3 respectively) and feed intake (1.30, 1.50 and 1.54 $\mathrm{kg} /$ day for T1, T2 and T3 respectively). However, feed conversion improved when ERT was added to the ration $(2.25,2.15$ and 2.04 for $\mathrm{T} 1, \mathrm{~T} 2$ and $\mathrm{T} 3$ respectively, $\mathrm{p}=0.012)$. The sensorial evaluation indicated that ERT inclusion in feed did not affect the taste of the meat. In conclusion, the inclusion up to $10 \%$ of ERT in pig feed improves feed conversion and do not affect the taste of meat.

Key words: pigs, trout silage, productive performance, feeding

\section{INTRODUCCIÓN}

El procesamiento de pescado genera entre 50 a $60 \%$ de residuos, los cuales consisten en cabezas, espinazos, aletas, pieles y vísceras. Estos residuos son altamente perecibles, por lo que requieren un adecuado manejo. En el Perú la extracción de trucha en el 2014 fue de 31401 toneladas métricas (INEI, 2016), de las cuales, cerca del 70\% correspondieron al cultivo en Puno, habiéndose generado alrededor de $10990 \mathrm{t}$ de residuos en esa región.

Una alternativa eficiente para el tratamiento y disposición de los residuos de pescado es la producción de ensilado para alimento animal (Ferraz de Arruda et al., 2007). La transformación de los residuos de pescado en ensilado y su posterior conservación se logra por fermentación al producir acidificación química, biológica o enzimática (Faid et al., 1994, 1997; Ferraz de Arruda et al., 2007). El ensilado de pescado es utilizado en reemplazo de la harina de pescado en la for- mulación de raciones para aves (Berenz et al., 1994; Betancourt et al., 2005; A1Marzooqi et al., 2010), peces (Barreto-Curiel et al., 2016) y cuyes (Mattos et al., 2003), con resultados satisfactorios.

Por otro lado, la producción eficiente de cerdos requiere de alimentos concentrados de alta calidad nutricional y digestibilidad, que permitan cubrir sus necesidades nutricionales. El ensilado de pescado ha sido utilizado en la formulación de alimentos para cerdos (Batterham et al., 1983; Kjos et al., 1999; Anuraj et al., 2014); sin embargo, se ha observado que niveles altos de inclusión en la ración producen sabor a pescado en la carne, reduciendo su aceptabilidad para el consumo humano (Cameron, 1962; Thuy et al., 2011).

La utilización de residuos de trucha en la elaboración de ensilado y su aplicación en la alimentación de cerdos no es una práctica común en la región de Puno, por lo que merece especial atención. Esta investigación fue realizada con el objetivo de determinar el efec- 
to del nivel de inclusión de ensilado de residuos de trucha en los alimentos de crecimiento y engorde sobre el consumo de alimento, ganancia de peso, conversión alimenticia de cerdos y su efecto en el sabor de la carne.

\section{Materiales y MéTodos}

\section{Medio Ambiente e Instalaciones}

La investigación se realizó en las instalaciones de la Empresa Arapa SAC, localizada en Iscayapi, provincia de Azángaro, a $105 \mathrm{~km}$ al norte de la ciudad de Puno. La zona se encuentra a $3880 \mathrm{~m}$ de altitud. Durante el experimento, la temperatura ambiental por la mañana fue de $12.4 \pm 2.0{ }^{\circ} \mathrm{C}$, al medio día de $25.0 \pm 4.5^{\circ} \mathrm{C}$ y por la tarde de $17.2 \pm 3.6^{\circ} \mathrm{C}$. La variación de la temperatura fue mayor a medio día, alcanzando una temperatura máxima de $31.2^{\circ} \mathrm{C}$, valor considerado muy alto para la altitud en que se desarrolló el estudio.

Se tuvieron 15 corrales de $6.1 \mathrm{~m}^{2}$ y 1.2 $m$ de altura. Estaban construidos en concreto, estando la mitad del área cubierto con techo de calamina. Los corrales estaban equipados con comederos fijos y bebederos tipo chupón.

\section{Animales y Manejo}

Se utilizaron 30 cerdos machos, sin castrar, de 45 días de edad, de línea comercial (Landrace-York-Duroc), provenientes de una granja comercial de Arequipa. Los cerdos tuvieron una semana de acostumbramiento en el lugar experimental y, seguidamente, fueron distribuidos al azar en tres tratamientos, según el nivel de ensilado de residuos de trucha (ERT) en el alimento: T1: $0 \%$, grupo testigo, y T2 con $5 \%$ y T3 con $10 \%$ de ERT en la ración. Cada tratamiento se replicó cinco veces ( 2 cerdos/réplica). El experimento duro 98 días y tanto el agua como el alimento fueron administrados ad libitum.

\section{Alimentos Experimentales}

Los ingredientes utilizados en las raciones se adquirieron en el mercado local, a excepción del ERT biológico, que se elaboró en las instalaciones de la empresa Arapa San Pedro y San Pablo SAC. con residuos del faenado de truchas de la planta de procesamiento. La técnica de fermentación del ERT fue desarrollada por el Instituto Tecnológico de la Producción a través de un trabajo de tesis con la utilización de «koji» (Aspergillus orizae), arroz y melaza (ChuracutipaMamani, 2016). El pH del ERT presentó valores menores o iguales a 4.5 y los valores de histamina presentaron valores menores a 85 $\mathrm{mg} / \mathrm{kg}$. El ERT contenía $61.3 \%$ de humedad, $12.0 \%$ de proteína bruta, $21.5 \%$ de grasa bruta y $5.3 \%$ de cenizas.

Se formularon seis raciones, siendo tres para crecimiento y tres para engorde (Cuadro 1). Se utilizó como referencia la tabla de composición de alimentos y requerimientos nutricionales de cerdos (NRC, 1998). Las raciones contenían 0,5 y $10 \%$ de ERT y contenían proporciones similares de proteína bruta (PB) y energía metabolizable (EM): $16.0 \%$ de PB y $3.20 \mathrm{Mcal} / \mathrm{kg}$ de EM para las dietas de crecimiento, y $13.0 \%$ de PB y $3.20 \mathrm{Mcal} / \mathrm{kg}$ de EM para las dietas de engorde. Fueron extruidas a $90{ }^{\circ} \mathrm{C}$ (Extrusor Khal ${ }^{\circledR} \mathrm{E} 8$, Alemania) en la planta de alimentos de la empresa arriba indicada. Las raciones fueron elaboradas cada 15 días y conservadas a $12{ }^{\circ} \mathrm{C}$ hasta su utilización.

El ERT se incluyó en la fórmula haciendo la equivalencia al $90 \%$ de materia seca (MS); es decir, $12.2 \%$ de ERT semilíquido, equivalente a $5 \%$ de inclusión en la ración, mientras que $24.3 \%$ de ERT semilíquido fue $10 \%$ de ERT en la ración.

\section{Análisis Químico}

Muestras de $500 \mathrm{~g}$ de cada ración experimental se empacaron al vacío y trasladadas al Laboratorio de Nutrición Animal de la 
Facultad de Medicina Veterinaria y Zootecnia de la Universidad Nacional del Altiplano (Puno, Perú), donde se mantuvieron en refrigeración $\left(3-4{ }^{\circ} \mathrm{C}\right)$ hasta su análisis.

Las muestras fueron analizadas por triplicado, siguiendo las recomendaciones de la AOAC (2011). La MS se determinó por el método gravimétrico. La PB por el método Kjeldahl (contenido de Nx 6.25). El contenido de grasa bruta por el método Soxhlet y la extracción se realizó con hexano. Las cenizas fueron determinadas por incineración de las muestras $\left(550^{\circ} \mathrm{C}\right.$ durante $\left.12 \mathrm{a} 20 \mathrm{~h}\right)$.

\section{Parámetros Productivos}

Se consideraron los periodos experimentales de crecimiento ( 0 a 56 días) y de engorde (57 a 98 días). El control del consumo de alimento se realizó a los 56 y 98 días del experimento con una balanza electrónica OHAUS ${ }^{\circledR}$ T32XW de $300 \pm 0.1 \mathrm{~kg}$ de capacidad. En ambos periodos experimentales, el consumo de alimento por día se determinó considerando el total de alimento consumido por periodo entre los días de alimentación (kg/día).

El peso vivo de los animales se determinó con una balanza electrónica $\mathrm{BOXA}^{\circledR}$ LP7510E de $3000 \pm 0.5 \mathrm{~kg}$ de capacidad. El control de peso se realizó a los 0 ( 45 días de edad), 56 y 98 días experimentales. El peso al inicio del experimento para los tratamientos fue de 13.1, 12.3 y $12.3 \mathrm{~kg}$, para T1, T2 y $\mathrm{T} 3$, respectivamente. La ganancia de peso por día se determinó considerando el peso vivo alcanzado en cada periodo entre los días experimentales $(\mathrm{kg} / \mathrm{dí})$.

La conversión alimenticia se determinó mediante la relación entre el consumo de alimento por día y la ganancia de peso por día de los cerdos en cada periodo. Es decir, el valor fue expresado en unidades de alimento utilizado para producir una unidad de peso vivo del animal (kg:kg).

\section{Beneficio}

Los cerdos fueron beneficiados al finalizar el experimento en la granja y en un mismo día. Se consideraron dos cerdos por tratamiento de $80 \mathrm{~kg}$ de peso vivo, aproximadamente, a fin de evitar el olor en la canal, que se presenta en machos sin castrar de mayor peso y edad. Los animales tuvieron un periodo de ayuno de 12 horas previo al beneficio, pero con acceso al agua ad libitum. La insensibilización se realizó por el método mecánico y la sangría por degüello. Las canales se mantuvieron en refrigeración a $4{ }^{\circ} \mathrm{C}$.

\section{Análisis Sensorial de la Carne}

La carne se dejó madurar durante 24 horas y luego fue almacenada bajo refrigeración a $5 \pm 1{ }^{\circ} \mathrm{C}$ en la planta de Arapa San Pedro y San Pablo SAC. Allí se separaron cerca de $4 \mathrm{~kg}$ de masa muscular (lomo y muslo) de cada cerdo, se trocearon y se hizo un pool; es decir $8 \mathrm{~kg}$ por tratamiento. Luego, la carne se separó en dos bolsas de $4 \mathrm{~kg}$ por tratamiento. Un grupo fue colocado en una caja de poliestireno expandido, con hielo, y enviada vía aérea a Lima. Las pruebas de evaluación se realizaron en el ITP (Lima) y en Arapa (Puno) el mismo día y a la misma hora.

Para cada tratamiento, la carne se cortó en trozos de $70 \mathrm{~g}$ y se realizó la cocción en agua con $1 \%$ de sal, a ebullición durante 45 min. Luego se retiró el agua y se continuó la cocción de la carne hasta dorar la parte externa por cerca de $15 \mathrm{~min}$. Los trozos de carne se presentaron a los evaluadores en platos con códigos que identificaban la procedencia de las muestras. Los evaluadores bebieron agua antes y luego de probar cada muestra.

El panel de evaluadores estuvo conformado por personas que consumen carne de cerdo, por lo menos una vez cada 15 días. Participaron 18 personas residentes en la ciu- 
Cuadro 1. Composición de las dietas experimentales para cerdos en crecimiento y engorde, según el porcentaje de ensilado de residuos de trucha (ERT)

\begin{tabular}{|c|c|c|c|c|c|c|}
\hline \multirow[t]{2}{*}{ Ingredientes } & \multicolumn{3}{|c|}{$\begin{array}{c}\text { Dietas de crecimiento }^{1} \\
\text { ERT }(\%)\end{array}$} & \multicolumn{3}{|c|}{$\begin{array}{c}\text { Dietas de engorde }^{2} \\
\text { ERT }(\%) \\
\end{array}$} \\
\hline & 0 & 5 & 10 & 0 & 5 & 10 \\
\hline Maíz amarillo molido & 56.37 & 53.52 & 52.68 & 54.36 & 60.00 & 60.00 \\
\hline Afrecho de trigo & 14.35 & 16.74 & 16.99 & 24.59 & 18.78 & 19.65 \\
\hline Harina de soya integral & 25.00 & 21.00 & 18.00 & 13.00 & 12.00 & 7.00 \\
\hline ERT (90\% MS) & 0.00 & 5.00 & 10.00 & 0.00 & 5.00 & 10.00 \\
\hline Aceite vegetal & 1.12 & 1.00 & 0.00 & 5.54 & 2.11 & 1.58 \\
\hline Fosfato bicálcico & 1.11 & 0.80 & 0.54 & 1.04 & 0.73 & 0.46 \\
\hline Lisina $\mathrm{HCl}$ & 0.20 & 0.14 & 0.07 & 0.05 & 0.01 & 0.01 \\
\hline Carbonato de calcio & 0.85 & 0.80 & 0.72 & 0.42 & 0.37 & 0.30 \\
\hline Montchack 3A-T3 & 0.50 & 0.50 & 0.50 & 0.50 & 0.50 & 0.50 \\
\hline Sal común & 0.40 & 0.40 & 0.40 & 0.40 & 0.40 & 0.40 \\
\hline $\begin{array}{l}\text { Premezcla (vitaminas }+ \\
\text { minerales) }\end{array}$ & 0.10 & 0.10 & 0.10 & 0.10 & 0.10 & 0.10 \\
\hline Total & 100.0 & 100.0 & 100.0 & 100.0 & 100.0 & 100.0 \\
\hline \multicolumn{7}{|l|}{ Nutrientes calculados: } \\
\hline $\begin{array}{l}\text { Energía metabolizable, } \\
\mathrm{Mcal} / \mathrm{kg}\end{array}$ & 3.20 & 3.20 & 3.20 & 3.20 & 3.20 & 3.20 \\
\hline Proteína cruda, \% & 16.00 & 16.06 & 16.34 & 13.00 & 13.68 & 13.41 \\
\hline Fibra cruda, $\%$ & 4.17 & 4.23 & 4.19 & 4.51 & 4.12 & 4.06 \\
\hline Calcio, $\%$ & 0.70 & 0.70 & 0.70 & 0.50 & 0.50 & 0.50 \\
\hline Fósforo disponible, $\%$ & 0.30 & 0.36 & 0.43 & 0.30 & 0.35 & 0.41 \\
\hline Lisina, $\%$ & 0.95 & 0.95 & 0.95 & 0.60 & 0.64 & 0.67 \\
\hline Metionina + Cistina & 0.54 & 0.54 & 0.55 & 0.43 & 0.47 & 0.47 \\
\hline Triptófano, \% & 0.21 & 0.20 & 0.20 & 0.18 & 0.17 & 0.16 \\
\hline Treonina, $\%$ & 0.65 & 0.65 & 0.68 & 0.50 & 0.56 & 0.56 \\
\hline \multicolumn{7}{|l|}{ Análisis proximal (en MS): } \\
\hline Materia seca, \% & 91.1 & 91.3 & 91.1 & 93.0 & 93.3 & 94.5 \\
\hline Proteína bruta, $\%$ & 15.9 & 15.9 & 15.6 & 13.5 & 14.5 & 14.4 \\
\hline Grasa bruta, $\%$ & 15.0 & 15.8 & 16.1 & 11.8 & 10.7 & 10.8 \\
\hline Cenizas, \% & 5.6 & 5.6 & 5.5 & 4.8 & 4.8 & 4.8 \\
\hline \multicolumn{7}{|c|}{$\begin{array}{l}{ }^{1} \text { A partir de los } 45 \text { días de edad } \\
{ }^{2} \text { A partir de los } 101 \text { días de edad } \\
{ }^{3} \text { Arcilla natural del altiplano peruano (Araníbar et al., 2012) } \\
{ }^{4} \text { Por cada kilogramo de alimento se adicionó } 9000 \text { UI de vit A, } 2000 \text { UI de vit D3, } 10 \text { UI de vit E, } 3 \mathrm{mg} \\
\text { de vit K, } 4 \mathrm{mg} \text { de riboflavina, } 10 \mathrm{mg} \text { de ácido pantoténico, } 30 \mathrm{mg} \text { de niacina, } 0.5 \mathrm{mg} \text { de ácido fólico, } \\
0.012 \mathrm{mg} \text { de vit B12, } 0.05 \mathrm{mg} \text { de biotina, } 60 \mathrm{mg} \text { de } \mathrm{Mn}, 40 \mathrm{mg} \text { de } \mathrm{Zn}, 80 \mathrm{mg} \text { de Fe, } 8 \mathrm{mg} \text { de Cu, } 1 \\
\text { mg de I, } 0.15 \mathrm{mg} \text { de Se, } 0.15 \mathrm{mg} \text { de Co, } 0.4 \mathrm{mg} \text { de butil hidroxitolueno (BHT) }\end{array}$} \\
\hline
\end{tabular}


Cuadro 2. Efecto del ensilado de residuos de trucha en la dieta (ERT) sobre el peso vivo $(\mathrm{kg})$ de los cerdos a los 56 y 98 días de iniciado el experimento

\begin{tabular}{|c|c|c|}
\hline Tratamiento & 56 días & 98 días \\
\hline Control & $43.4^{\mathrm{a}}$ & $69.8^{\mathrm{a}}$ \\
\hline ERT, $5 \%$ & $43.0^{\mathrm{a}}$ & $81.3^{\mathrm{a}}$ \\
\hline ERT, $10 \%$ & $44.0^{\mathrm{a}}$ & $86.4^{\mathrm{a}}$ \\
\hline $\operatorname{EEM}^{1}(n=5)$ & 1.64 & 2.43 \\
\hline Probabilidad & 0.227 & 0.224 \\
\hline \multicolumn{3}{|c|}{$\begin{array}{l}\text { Error estándar de la media, } 5 \\
\text { repeticiones por media } \\
\text { No hubo diferencia estadística dentro } \\
\text { de días experimentales entre trata- } \\
\text { mientos }\end{array}$} \\
\hline
\end{tabular}

dad de Lima y con edades entre 35 y 55 años, así como 18 personas residentes en Puno, con edades entre 20 y 65 años. Los evaluadores fueron informados de las características de la ficha sensorial; además, se les pidió que anotaran la percepción de posibles sabores anormales.

La prueba sensorial tuvo como propósito establecer posibles diferencias en el gusto entre las muestras, así como la magnitud de estas diferencias. Para esto, se aplicó una modificación a la prueba de comparaciones apareadas de Scheffé (Anzaldúa-Morales, 1994). Las muestras de T1 (testigo) fueron comparadas con los tratamientos T2 y T3 en una escala de siete puntos, donde 7: sabor mucho mejor que la muestra T; 6 : sabor moderadamente mejor que la muestra $\mathrm{T} 1 ; 5$ : sabor levemente mejor que la muestra T1; 4 : sabor igual que la muestra $\mathrm{T} 1 ; 3$ : sabor levemente peor que la muestra $\mathrm{T} 1 ; 2$ : sabor moderadamente peor que la muestra $\mathrm{T} 1 ; 1$ : sabor mucho peor que la muestra $\mathrm{T} 1$.

Los evaluadores probaron una porción de la muestra testigo (T1) y la compararon con la muestra $\mathrm{T} 2$, tomando entre 3 a $5 \mathrm{mi}$ - nutos para la evaluación. Descansaron cerca de 5 minutos, bebieron medio vaso de agua tibia e hicieron la comparación para $\mathrm{T} 1$ con T3. Los evaluadores desconocían la procedencia o tratamiento de las muestras. La temperatura de la carne en el momento de la degustación estuvo entre 60 y $65^{\circ} \mathrm{C}$.

\section{Análisis Estadístico}

El análisis estadístico fue realizado usando el procedimiento de modelo lineal general (GLM) del programa estadístico SAS (2004). Los datos de peso vivo, ganancia de peso, consumo de alimento y conversión alimenticia se sometieron al análisis de medias por mínimos cuadrados (LS Means) en un diseño completamente al azar (DCA) con tres tratamientos y cinco repeticiones por tratamiento (Littell et al., 2002). Los resultados son expresados por medias y error estándar de la media (EEM).

Para el análisis de sabor de la carne se usó el Programa Minitab ${ }^{\circledR}$, donde se evaluó la homogeneidad de varianza y normalidad de los resultados y, luego de cumplir los supuestos, se aplicó el análisis de varianza a los resultados. Las diferencias fueron consideradas estadísticamente significantes cuando $\mathrm{p}<0.05$.

\section{Resultados y Discusión}

El control de peso vivo a los 56 días para el grupo de crecimiento y a los 98 días para el grupo de engorde se muestra en el Cuadro 2. Se observa una diferencia biológica para los cerdos del tratamiento con $10 \%$ de ERT a los 98 días; sin embargo, las diferencias no fueron estadísticamente significativas.

En el Cuadro 3 se aprecia que no hubo diferencias significativas en la ganancia diaria de peso. En el periodo de crecimiento ( 0 a 56 días) se observó un mayor consumo de alimento en T2 (1.06 kg/día) y T3 (1.07 kg/día) 
Cuadro 3. Efecto del ensilado de residuos de trucha (ERT) en la dieta sobre la ganancia diaria de peso, consumo diario de alimento e índice de conversión alimenticia en cerdos durante 98 días de suplementación

\begin{tabular}{lccc}
\hline Tratamientos & 0 a 56 días & 57 a 98 días & 0 a 98 días \\
\hline Ganancia de peso por día (kg/día) & & & \\
Control & 0.55 & 0.63 & 0.59 \\
ERT, 5\% & 0.54 & 0.91 & 0.70 \\
ERT, 10\% & 0.56 & 1.01 & 0.75 \\
EEM ${ }^{1}(\mathrm{n}=5)$ & 0.030 & 0.073 & 0.025 \\
Probabilidad & 0.428 & 0.314 & 0.237 \\
Consumo de alimento por día (kg/día) & & \\
Control & $0.94^{\mathrm{b}}$ & 1.80 & 1.30 \\
ERT, 5\% & $1.06^{\mathrm{a}}$ & 2.10 & 1.50 \\
ERT, 10\% & $1.07^{\mathrm{a}}$ & 2.16 & 1.54 \\
EEM (n=5) & 0.042 & 0.104 & 0.042 \\
Probabilidad & 0.043 & 0.831 & 0.149 \\
Índice de conversión alimenticia (kg:kg) & & \\
Control & 1.72 & $2.87^{\mathrm{a}}$ & $2.25^{\mathrm{a}}$ \\
ERT, 5\% & 1.96 & $2.33^{\mathrm{b}}$ & $2.15^{\mathrm{a}}$ \\
ERT, 10\% & 1.92 & $2.14^{\mathrm{b}}$ & $2.04^{\mathrm{b}}$ \\
EEM (n=5) & 0.059 & 0.089 & 0.042 \\
Probabilidad & 0.288 & 0.017 & 0.012 \\
\hline
\end{tabular}

${ }^{1}$ Error estándar de la media, 5 repeticiones por media

${ }^{a, b}$ Medias con letras distintas en la misma columna son diferentes a la Prueba de LSMeans $(\mathrm{p}<0.05)$

Cuadro 4. Calificaciones sensoriales de sabor de la carne de cerdo

\begin{tabular}{lll}
\hline Evaluadores & ERT 5\% & ERT 10\% \\
\hline ITP & $5.0 \pm 1.2$ & $4.8 \pm 1.1$ \\
Arapa & $5.1 \pm 1.4$ & $4.6 \pm 1.4$ \\
\hline
\end{tabular}

con relación a $\mathrm{T} 1(0.94 \mathrm{~kg} / \mathrm{día})(\mathrm{p}<0.05)$; sin embargo, este efecto no perduró en forma significativa en el periodo de engorde. Tampoco se apreciaron las diferencias cuando se consideró el total del periodo experimental ( 0 98 días).

La conversión alimenticia mejoró con el incremento de inclusión de ERT en la ración en el periodo de engorde $(\mathrm{p}=0.017)$. Así, para el grupo control fue de 2.25 y para el grupo de $5 \%$ correspondió a 2.15 , mientras que los que recibieron $10 \%$ de ERT en la ración fue significativamente mejor $(2.04)(\mathrm{p}<0.05)$. Estos resultados concuerdan con los reportados por Batterham et al. (1983), quienes reportaron mejoras en las conversiones alimenticias con el incremento de ensilado en la 
ración de cerdos alimentados. Por otro lado, Areche et al. (1994), utilizando niveles de 10, 20 y $30 \%$ de ensilado fresco, obtuvieron mayor incremento de peso y mejor índice de conversión alimenticia con el incremento de ensilado en la ración de cerdos, en tanto que Thuy et al. (2011) reportaron mayor ganancia diaria de peso $(0.76 \mathrm{~kg} / \mathrm{día})$ y mejor conversión alimenticia (3.15) en cerdos sustituyendo el $100 \%$ de harina de pescado por ensilado de pescado (Pangasius hypophthalmus). Esos resultados difieren a los obtenidos en el presente trabajo y podrían ser atribuidos a la metodología utilizada, así como al distinto proceso de ensilado.

Contrariamente, Kjos et al. (1999) no encontraron diferencias en el rendimiento productivo utilizando $5 \%$ de ensilado de salmón con tres niveles de grasa de pescado $(0.25,0.50,0.95 \%)$. Asimismo, Tibbets et al. (1981), utilizando ensilado de pescado y mariscos (cangrejos, camaroncillos, estrellas de mar, tiburones, corvina, pescadillas, merluzas y cabezas de camarones) encontraron que niveles altos de inclusión ( 6 y 9\%) en la ración desmejoraron la conversión alimenticia (3.51 y 3.80$)$ en comparación con niveles bajos (0 y $3 \%)$, donde hubo una mejor respuesta $(3.13$ y 3.30$)$.

Los análisis químicos indicaron una alta digestibilidad in vitro de la proteína en las raciones con 5 y $10 \%$ de ERT ( 89.3 y $89.2 \%$, respectivamente). Nørgaard et al. (2015) reportan que la digestibilidad ileal de la proteína del ensilado de residuos de salmón en cerdos fue $61 \%$ y la digestibilidad de los aminoácidos esenciales (78\%) es mayor a los no esenciales (47\%). Asimismo, Borghesi (2004), citado por Ferraz de Arruda et al (2007) afirman que la digestibilidad de la proteína cruda del ensilado biológico de residuos de tilapia fue alta (89.1\%).

El ERT utilizado en el presente estudio con una equivalencia al estado fresco de 12.5 y $24.3 \%$ de inclusión en las dietas de crecimiento y engorde, no afectaron el sabor $\mathrm{u}$ olor de la carne. Las calificaciones de sabor de la carne de cerdo evaluadas en ITP, Lima y Arapa, Puno, no presentaron diferencia significativa entre ellos (Cuadro 4). Las calificaciones promedio de las muestras fueron mayores a 4 , indicando que los tratamientos evaluados presentaron mejor sabor que el testigo (T1).

Al-Marzooqui et al. (2010) mencionan que al remplazar la soya por una mezcla de ensilado de pescado de 10 y $20 \%$ y maíz (85:15) en la dieta no se detectó sabor a pescado en la carne de pollo. Asimismo, Tibbets et al. (1981) indicaron que niveles de inclusión de 3, 6 y 9\% de ensilado mixto de pescado y mariscos en la ración no produjeron cambios en el sabor de la carne de cerdos.

\section{Conclusiones}

- La inclusión de 5 y $10 \%$ de ensilado de residuos de trucha en la ración no afectaron el consumo de alimento ni el peso vivo de los cerdos, pero, el índice de conversión alimenticia mejoró con el nivel de $10 \%$ de ensilado.

- La inclusión de 5 y $10 \%$ de ensilado de residuos de trucha en el alimento de cerdos en crecimiento y engorde no produjeron cambios en el sabor de la carne de los cerdos.

\section{Agradecimientos}

Los autores agradecen a INNOVATE PERÚ por el financiamiento de este proyecto con código PIPEI-6-P-024-005-13.

\section{Literatura Citada}

1. Anzaldúa-Morales A. 1994. La evaluación sensorial de los alimentos en la teoría y la práctica. Zaragoza, España: Acribia. 198 p.

2. Araníbar MJ, Mateos GG, Mamani F, Portocarrero H, Arizabal J, Jara C, Oblitas W, et al. 2012. Arcillas comestibles del altiplano peruano-boliviano. 
Puno, Perú: Universidad Nacional del Altiplano. $37 \mathrm{p}$.

3. $A O A C$. 2011. Association of Official Analytical Chemists. Official methods of analysis. $18^{\text {th }}$ ed. Gaithersburg, MD, USA; AOAC.

4. Al-Marzooqui W, Al-Farsi MA, Kadim IT, Mahgoub O, Goddard JS. 2010. The effect of feeding different levels of sardine fish silage on broiler performance, meat quality and sensory characteristics under closed and open-sided housing systems. Asian Australs J Anim Sci 23: 1614-1625. doi: 10.5713/ ajas.2010.10119

5. Anuraj KS, Shyama K, Sreeparvathy M, Ajith MK. 2014. Effect of dietary incorporation of dried tuna waste silage on nutrient digestibility in large white Yorkshire pigs. Int J Food Nutr Sci 3: 211-213.

6. Areche N, Berenz Z, León G. 1994. Utilización del ensilado de residuos de pescado en dietas para cerdos. Bol Inv Inst Tec Pes 4(1): 77-90.

7. Barreto-Curiel F, Parés-Sierra G, Correa-Reyes G, Durazo-Beltrán E, Viana MT. 2016. Total and partial fishmeal substitution by poultry byproduct meal (petfood grade) and enrichment with acid fish silage in aquafeeds for juveniles of rainbow trout Oncorhynchus mykiss. Lat Am J Aquat Res 44: 327-335. doi: 10.3856/vol44issue2-fulltext-13

8. Batterham ES, Gorman TBS, Chvojka

$R .1983$. Nutritional value and mercury content of fish silage for growing pigs. Anim Feed Sci Technol 9: 169-179. doi: 10.1016/0377-8401(83)90031-7

9. Berenz Z, Romero F, Beoutis G 1994. Utilización del ensilado de residuos de pescado en dietas para pollos de carne. Bol Inv Inst Tec Pes 4(1): 91-104.

10. Betancourt L, Díaz GJ, Aguilar X, Ríos J. 2005. Efecto del ensilaje de vísceras de trucha (Oncorhynchus mykiss) sobre el comportamiento productivo y el contenido de ácidos omega-3 en hígado, muslos y pechuga, de pollos de engorde.
Livest Res Rural Develop 17(9). [Internet]. Disponible en: http:// www.lrrd.org/lrrd17/9/beta17106.htm

11. Cameron CDT. 1962. Acid fish offal silage as a course of protein in growing and finishing rations for bacon pigs. Canadian J Anim Sci 42: 41-48. doi: 10.4141/cjas62-006

12. Churacutipa-Mamani M. 2016. Obtención de un ensilado biológico a partir de residuos de trucha (Oncorhynchus mykiss). Tesis de Ingeniero Agroindustrial. Puno, Perú : Univ Nacional del Altiplano.

13. Faid M, Karani H, Elmarrakchi A, Achkari-Begdouri A. 1994. A biotechnological process for the valorization of fish waste. Bioresource Technol 49: 237-241. doi: 10.1016/09608524(94)90046-9

14. Faid M, Zouiten A, Elmarrakchi A, Achkari-Begdouri A. 1997. Biotransformation of fish waste into a stable feed ingredient. Food Chem 60: 13-18. doi: 10.1016/S0308-8146(96)00291-9

15. Ferraz de Arruda L, Borghesi R, Oetterer M. 2007. Use of fish waste as silage - a review. Braz Arch Biol Technol 50: 879-886. doi: 10.1590/S151689132007000500016

16. [INEI] Instituto Nacional de Estadística e Informática. 2016. Series nacionales. Extracción de trucha fresca. [Internet]. Disponible en: http:// series.inei.gob.pe:8080/sirtod-series/

17. Kjos NP, A. Skrede A, Overland M. 1999. Effects of dietary fish silage and fish fat on growth performance and sensory quality of growing-finishing pigs. Canadian J Anim Sci 79: 139-147. doi: 10.4141/A98-091

18. Littell RC, Stroup WW, Freund RJ, 2002. SAS for linear models. $4^{\text {th }}$ ed. USA: SAS Inst. Cary, NC. 329 p.

19. Mattos CJ, Chauca FL, San Martín F, Carcelén CF, Arbaiza FT. 2003. Uso del ensilado biológico de pescado en la alimentación de cuyes mejorados. Rev Inv Vet Perú 14: 89-96. doi: 10.15381/rivep.v14i2.1612 
20. Norgaard JV, Petersen JK, Torring DB, Jorgensen H, Larke HN. 2015. Chemical composition and standardized ileal digestibility of protein and amino acids from blue mussel, starfish, and fish silage in pigs. Anim Feed Sci Technol 205: 90-97. doi: 10.1016/j.anifeedsci.2015.04.005

21. NRC. 1998. Nutrient requirements of swine. $10^{\text {th }}$ ed. Washington, DC, USA: National Academy Press. 210 p.

22. Tibbets GW, Seerley RW, McCampbell HC, Vezey $S A$. 1981. An evaluation of an ensiled waste fish product in swine diets. J Anim Sci 52: 93-100. doi:10.2527/ jas1981.52193x

23. Thuy NT, Lindberg JE, Ogle B. 2011. Effects of replacing fish meal with ensiled catfish (Pangasius hypophthalmus) by-products on the performance and carcass quality of finishing pigs. $\mathrm{J}$ Anim Feed Sci 20: 47-59.

24. SAS Institute Inc. 2004. SAS/STAT ${ }^{\circledR}$ 9.1 User's guide. Cary, NC, USA: SAS Institute. $5121 \mathrm{p}$ 